\title{
Impact of pulmonary thromboendarterectomy on tricuspid regurgitation in patients with chronic thromboembolic pulmonary hypertension: a single-center prospective cohort experience
}

\author{
Yanan Zhen ${ }^{1 \#}$, Jianbin Zhang ${ }^{1 \#}$, Xiaopeng Liu ${ }^{1}$, Guang Sun ${ }^{1}$, Xia Zheng ${ }^{1}$, Yongxin Han ${ }^{1}$, Zhenguo Zhai ${ }^{2}$, \\ Aili Li ${ }^{3}$, Fan Lin ${ }^{1}$, Peng Liu ${ }^{1}$ \\ ${ }^{1}$ Department of Cardiovascular Surgery, ${ }^{2}$ Department of Respiratory and Critical Care Medicine, Center of Respiratory Medicine, ${ }^{3}$ Department of \\ Ultrasonic Medicine, China-Japan Friendship Hospital, Beijing 100029, China \\ Contributions: (I) Conception and design: Y Zhen, J Zhang, P Liu; (II) Administrative support: P Liu; (III) Provision of study materials or patients: X \\ Liu, G Sun; (IV) Collection and assembly of data: X Zheng, Y Han; (V) Data analysis and interpretation: Z Zhai, A Li; (VI) Manuscript writing: All \\ authors; (VII) Final approval of manuscript: All authors. \\ \#These authors contributed equally to this work. \\ Correspondence to: Peng Liu. Department of Cardiovascular Surgery, China-Japan Friendship Hospital, Beijing 100029, China. \\ Email: Liupeng6618@yeah.net.
}

Background: For patients with chronic thromboembolic pulmonary hypertension (CTEPH) and tricuspid regurgitation (TR) undergoing pulmonary thromboendarterectomy (PTE), whether concomitant tricuspid annuloplasty should be carried out is still controversial.

Methods: The study population consisted of 45 consecutive patients with CTEPH who were scheduled to undergo PTE. All PTE surgeries were conducted with a median sternotomy and deep hypothermia circulatory arrest (DHCA). We collected and analyzed the demographics, surgical details, echocardiographic parameters, and right heart catheterization (RHC) results of these patients.

Results: Moderate to severe TR was documented in 48.9\% (22/45) of the patients pre-operatively and $4.4 \%$ (2/45) of the patients post-operatively. In patients with grade $4 \mathrm{TR}$, severity decreased to grade 2 in 8 and to grade 1 in 1 . In patients with grade $3 \mathrm{TR}$, severity decreased to grade 2 in 9 , to grade 1 in 3 , and 1 remained unchanged. In patients with grade 2 TR, severity decreased to grade 1 in 8 , and 15 remained unchanged. The post-operative TR velocity was decreased significantly $(431.9 \pm 53.4$ vs. $196.5 \pm 154.0, \mathrm{P}<0.001)$. Pulmonary artery systolic pressure was $84 \pm 17 \mathrm{mmHg}$ pre-operatively and decreased to $38 \pm 14 \mathrm{mmHg}$ post-operatively $(\mathrm{P}<0.001)$. The pre and post-operative pulmonary diastolic pressure was $29 \pm 9$ and $17 \pm 7 \mathrm{mmHg}$, respectively $(\mathrm{P}<0.001)$. The pre and post-operative mean pulmonary pressure was $48 \pm 10$ and $24 \pm 9 \mathrm{mmHg}$, respectively $(\mathrm{P}<0.001)$. The pulmonary vascular resistance $(\mathrm{PVR})\left(1,025.4 \pm 465.0\right.$ vs. $476.6 \pm 181.2$ dynes $\left.\cdot \mathrm{sec}^{\mathrm{cm}} \mathrm{cm}^{-5}, \mathrm{P}<0.001\right)$ and pulmonary artery wedge pressure (PAWP) $(9 \pm 4$ vs. $5 \pm 2 \mathrm{mmHg}, \mathrm{P}<0.001)$ decreased significantly after operation. The cardiac index (CI) increased significantly $(1.9 \pm 0.5$ vs. $2.3 \pm 0.4, \mathrm{P}=0.003)$ after operation.

Conclusions: In conclusion, functional TR could be alleviated after PTE even in patients with high PVR. However, the long-term results need to be further investigated.

Keywords: Pulmonary thromboendarterectomy (PTE); chronic thromboembolic pulmonary hypertension (CTEPH); tricuspid regurgitation (TR)

Submitted Oct 03, 2019. Accepted for publication Dec 10, 2019.

doi: $10.21037 /$ jtd.2019.12.99

View this article at: http://dx.doi.org/10.21037/jtd.2019.12.99 


\section{Introduction}

Chronic thromboembolic pulmonary hypertension (CTEPH) develops in $0.56-3.8 \%$ of acute pulmonary embolism patients $(1,2)$, and is characterized by intraluminal thrombus organization, fibrosis, and subsequent unobstructed small vessel remodeling (3). These characterizations result in pulmonary hypertension and progressive right heart failure (4).

Sustained pulmonary hypertension may lead to persistent right ventricle pressure overload, tricuspid annular dilatation, and regurgitation. Such functional tricuspid regurgitation (TR) also occurs in advanced mitral valve disease. For patients with severe functional TR caused by mitral valve disease and pulmonary hypertension, mitral valve surgery with simultaneous tricuspid annuloplasty is safe, effective, and associated with better long-term rightsided remodeling (5). However, for severe functional TR caused by CTEPH, some studies have shown there to be a significant improvement of tricuspid valve function after pulmonary thromboendarterectomy (PTE) without simultaneous tricuspid annuloplasty $(6,7)$. But the enrolled patients were not very severe, the pulmonary vascular resistance (PVR) was relatively not very high. In China, most of the patients undergoing PTE surgery were advanced-stage CTEPH with $>1,000$ dynes $\cdot \mathrm{sec} \cdot \mathrm{cm}^{-5} \mathrm{PVR}$, maybe because the awareness and understanding for the disease is still lacking.

For patients with relatively advanced-stage CTEPH and TR who undergo PTE, whether or not tricuspid annuloplasty should simultaneously be conducted is still controversial. Our study aimed to review our data of PTE without simultaneous tricuspid annuloplasty and evaluate the impacts on the changes in pulmonary artery pressure, $\mathrm{TR}$, and right heart function.

\section{Methods}

\section{Study population}

The study population consisted of 45 consecutive patients with CTEPH who were scheduled to undergo PTE between December 2016 and June 2019 at our hospital. Patients who did not undergo echocardiography preoperatively or within one month post-operatively were excluded. We collected and analyzed the demographics, surgical details, echocardiographic parameters, and right heart catheterization (RHC) results of these patients. The study procedures followed institutional guidelines and was approved by the institutional review board of our hospital. The enrolled patients have signed the informed consent for the use of the data. All of the data was collected prospectively. The report of this study followed Strengthening the Reporting of Cohort Studies (STROCSS) criteria.

\section{Surgical procedure}

All PTE surgeries were conducted with a median sternotomy and deep hypothermia circulatory arrest (DHCA). The technique of the procedure was in accordance with that reported by the university of California San Diego Health Center (8). Circulatory arrest time was strictly limited to 20 minutes; when more time was needed, we restarted the cardiopulmonary bypass (CPB) for 10 minutes, and then PTE was continued with a second circulatory arrest. After complete thromboendarterectomy, we sutured the pulmonary artery. We then rewarmed the patients, stopped CPB, and closed the incision. Patients were transferred to the intensive care unit (ICU) after the procedure.

\section{Transthoracic echocardiographic parameters}

Patients underwent transthoracic echocardiography before and within one month after PTE surgery. All echocardiograms were performed with the commercially available machine, EPIQ 7C (Philips, USA), with a 1.0-5.0 MHz linear array transducer S5-1. Patients were in left-lateral position, and II lead electrocardiogram was monitored.

Pulmonary artery diameter (PAD), pulmonary artery velocity (PAV), transverse diameter of right atrium (TDRA), transverse diameter of right ventricular basal segment, and transverse diameter of left ventricle were measured. The area of TR was determined in the apical four-chamber view according to the following criteria: grade 1 (trivial or no TR, $0<$ jet area $<2 \mathrm{~cm}^{2}$ ), grade 2 (mild TR, $2<$ jet area $<4 \mathrm{~cm}^{2}$ ), grade 3 (moderate TR, $4<$ jet area $<10 \mathrm{~cm}^{2}$ ), and grade 4 (severe TR, jet area $>10 \mathrm{~cm}^{2}$ ). All data was stored digitally for further analysis. Two independent investigators did the analysis and the investigators were blinded to whether the images were from before or after surgery. All measurements were in accordance with the guidelines for echocardiographic assessment (9).

\section{RHC}

RHC was performed for the included patients using 


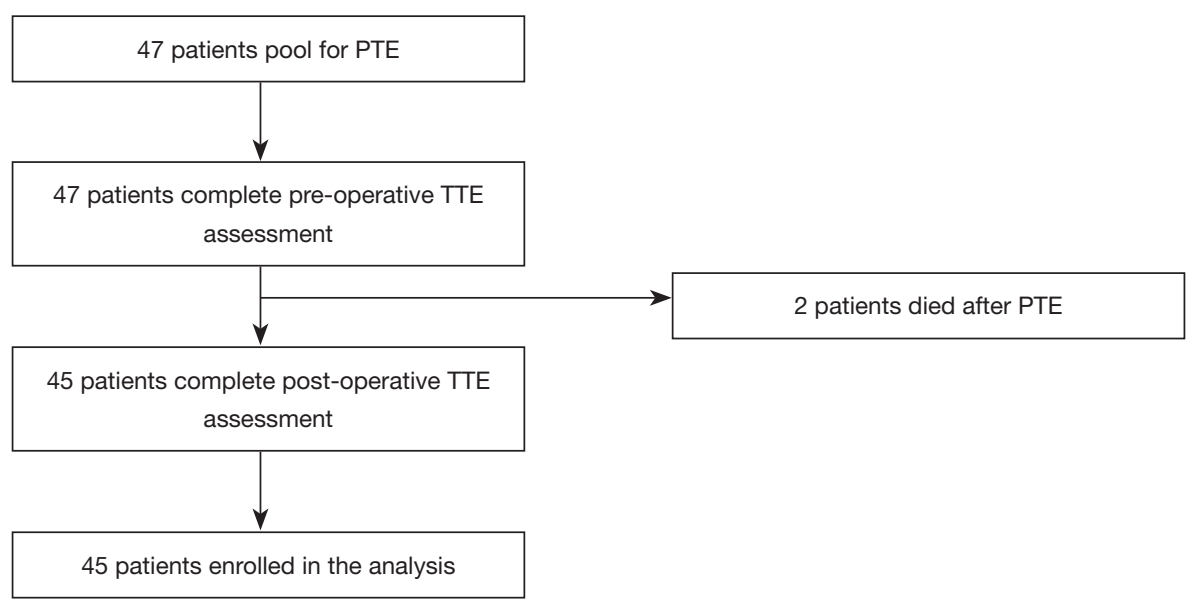

Figure 1 Flow diagram of the study. PTE, pulmonary thromboendarterectomy; TTE, transthoracic echocardiography.

a Swan-Ganz catheter through a femoral vein access. Pulmonary artery pressure (systolic, diastolic, and mean), PVR, pulmonary artery wedge pressure (PAWP), and cardiac index (CI) were measured with the commercially available machine Vigileo (Edward, USA).

\section{Statistical analyses}

Continuous variables were presented as mean $\pm \mathrm{SD}$, and discrete variables were presented as numbers and percentages. A two-sided paired student $t$-test was performed for continuous variables, and the chi-square test or Fisher's exact test was used to analyze discrete variables. Multivariate stepwise logistic regression analysis was applied to detect independent predictors of significant TR. The pre-operative and post-operative TR grade comparison was conducted using Wilcoxon rank-sum test. Spearman's correlation coefficient was used to evaluate the correlation between pre-operative, post-operative TR, and other variables. Data analysis was performed using SPSS version 23 (SPSS Inc., Chicago, IL, USA). A P value of $<0.05$ was considered statistically significant.

\section{Results}

\section{Baseline characteristics}

Finally, we enrolled 45 CTEPH patients (28 males; mean age $49 \pm 12$ years) who received PTE surgery with DHCA (Figure 1). The duration of symptomatic pulmonary hypertension was $5.0 \pm 4.6$ years. New York Heart Association (NYHA) functional classification was recorded
Table 1 Demographics and clinical features

\begin{tabular}{lc}
\hline Characteristics & No. \\
\hline Age (years) & $49 \pm 12$ \\
Gender (men/women) & $28 / 17$ \\
BMI & $23.7 \pm 3.7$ \\
Duration of symptomatic pulmonary & $5.0 \pm 4.6$ \\
hypertension (years) & \\
NYHA classification (n) & \\
2 & 10 \\
3 & 24 \\
4 & 11 \\
Commodities (n) & \\
Coronary artery disease & 1 \\
Deep venous thrombosis & 20 \\
Antiphospholipid syndrome & 6 \\
Hyperhomocysteinemia & 6 \\
Hereditary antithrombin III deficiency & 1 \\
\hline BMl, body mass index; NYHA, New Yor Heat AssOciation. & \\
\hline
\end{tabular}

BMI, body mass index; NYHA, New York Heart Association.

for every patient. The demographics and clinical features are shown in Table 1.

\section{RHC}

The results of pre-operative and post-operative RHC are shown in Table 2. Pulmonary artery systolic pressure was $84 \pm 17 \mathrm{mmHg}$ pre-operatively and decreased to $38 \pm 14$ 
Table 2 The results of pre-operative and post-operative right heart catheterization

\begin{tabular}{|c|c|c|c|}
\hline Variable & Pre-operative & Post-operative & $P$ value \\
\hline dPAP (mmHg) & $29 \pm 9$ & $17 \pm 7$ & $<0.001$ \\
\hline mPAP $(\mathrm{mmHg})$ & $48 \pm 10$ & $24 \pm 9$ & $<0.001$ \\
\hline $\mathrm{Cl}\left[\mathrm{L} /\left(\min \cdot \mathrm{m}^{2}\right)\right]$ & $1.9 \pm 0.5$ & $2.3 \pm 0.4$ & 0.003 \\
\hline PAWP (mmHg) & $9 \pm 4$ & $5 \pm 2$ & $<0.001$ \\
\hline
\end{tabular}

SPAP, systolic pulmonary artery pressure; dPAP, diastolic pulmonary artery pressure; mPAP, mean pulmonary artery pressure; Cl, cardiac index; PVR, pulmonary vascular resistance; PAWP, pulmonary artery wedge pressure.

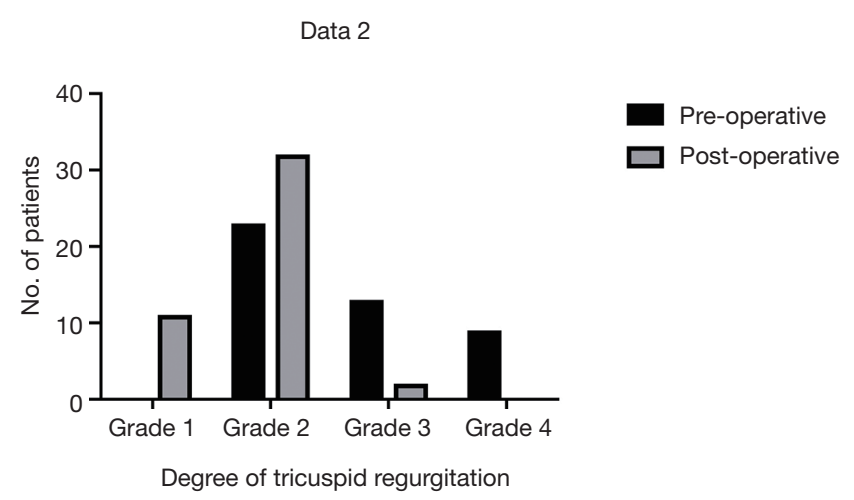

Figure 2 The pre-operative and post-operative severity of tricuspid regurgitation.

mmHg post-operatively $(\mathrm{P}<0.001)$. The pre and postoperative pulmonary diastolic pressure was $29 \pm 9$ and $17 \pm 7 \mathrm{mmHg}$, respectively $(\mathrm{P}<0.001)$. The pre-operative and post-operative mean pulmonary pressure was $48 \pm 10$ and $24 \pm 9 \mathrm{mmHg}$, respectively $(\mathrm{P}<0.001)$. The PVR $\left(1,025.4 \pm 465.0\right.$ vs. $476.6 \pm 181.2$ dynes $\left.\cdot \mathrm{sec} \cdot \mathrm{cm}^{-5}, \mathrm{P}<0.001\right)$ and PAWP $(9 \pm 4$ vs. $5 \pm 2 \mathrm{mmHg}, \mathrm{P}<0.001)$ was decreased significantly after operation. CI was increased significantly $\left[1.9 \pm 0.5\right.$ vs. $\left.2.3 \pm 0.4 \mathrm{~L} /\left(\mathrm{min} \cdot \mathrm{m}^{2}\right), \mathrm{P}=0.003\right]$ after operation.

\section{Severity of TR}

Moderate to severe TR was documented in $48.9 \%$ (22/45) of patients pre-operatively and in $4.4 \%(2 / 45)$ of patients postoperatively (Figure 2). In patients with grade 4 TR, severity decreased to grade 2 in 8 and to grade 1 in 1 . In patients with grade 3 TR, severity decreased to grade 2 in 9 , to grade 1 in 3 , and 1 remained unchanged. In patients with grade $2 \mathrm{TR}$, severity decreased to grade 1 in 8 , and 15 remained unchanged. The post-operative TR velocity was decreased significantly $(431.9 \pm 53.4 v s .196 .5 \pm 154.0 \mathrm{~cm} / \mathrm{sec}, \mathrm{P}<0.001)$.

\section{Right ventricular and tricuspid morphology}

The results of pre-operative and post-operative cardiac ultrasonography are shown in Table 3. The pre-operative $\mathrm{PAD}$ was $33.7 \pm 5.2 \mathrm{~mm}$ and decreased to $29.1 \pm 6.1 \mathrm{~mm}$ postoperatively $(\mathrm{P}=0.003)$. The pre-operative and post-operative PAV were $94.3 \pm 69.3$ and $92.0 \pm 30.0 \mathrm{~cm} / \mathrm{sec}$, respectively $(\mathrm{P}=0.840)$. The TDRA was decreased from $50.4 \pm 9.1$ to $41.4 \pm 6.5 \mathrm{~mm}(\mathrm{P}<0.001)$.

\section{Correlation of pre-operative, post-operative TR, and other variables}

Pre-operative TR was correlated with $\mathrm{mPAP}(\mathrm{r}=0.340$, $\mathrm{P}=0.029)$, PVR ( $\mathrm{r}=0.392, \mathrm{P}=0.018)$, and TDRA $(\mathrm{r}=0.758$, $\mathrm{P}<0.001)$. Post-operative TR has reversed correlation with post-operative $\mathrm{CI}(\mathrm{r}=-0.298, \mathrm{P}=0.047)$.

\section{Discussion}

CTEPH is a progressive pulmonary vascular disease. This disease is characterized by a direct organized embolic occlusion and the remodeling of larger pulmonary arteries. Later, small vessel disease is caused by unresolved thrombotic materials (10). Persistent pulmonary hypertension and right ventricle overload cause right ventricular hypertrophy, accompanied by tricuspid annular dilatation and subsequent functional TR (11). Menzel et al. (12) reported $89.7 \%$ CTEPH patients have moderate to severe TR while Thistlethwaite et al. (13) reported a mean TR of $3+$ (TR jet filling more than $66 \%$ of the 
Table 3 The results of pre-operative and post-operative cardiac ultrasonography

\begin{tabular}{|c|c|c|c|}
\hline Variable & Pre-operative & Post-operative & $P$ value \\
\hline Grade 1 & 0 & 11 & \\
\hline Grade 2 & 23 & 32 & \\
\hline Grade 3 & 13 & 2 & \\
\hline TR velocity $(\mathrm{cm} / \mathrm{sec})$ & $431.9 \pm 53.4$ & $196.5 \pm 154.0$ & $<0.001$ \\
\hline PAD $(m m)$ & $33.7 \pm 5.2$ & $29.1 \pm 6.1$ & 0.003 \\
\hline $\mathrm{PAV}(\mathrm{cm} / \mathrm{sec})$ & $94.3 \pm 69.3$ & $92.0 \pm 30.0$ & 0.840 \\
\hline TDRA (mm) & $50.4 \pm 9.1$ & $41.4 \pm 6.5$ & $<0.001$ \\
\hline
\end{tabular}

TR, tricuspid regurgitation; PAD, pulmonary artery diameter; PAV, pulmonary artery velocity; TDRA, transverse diameter of the right atrium.
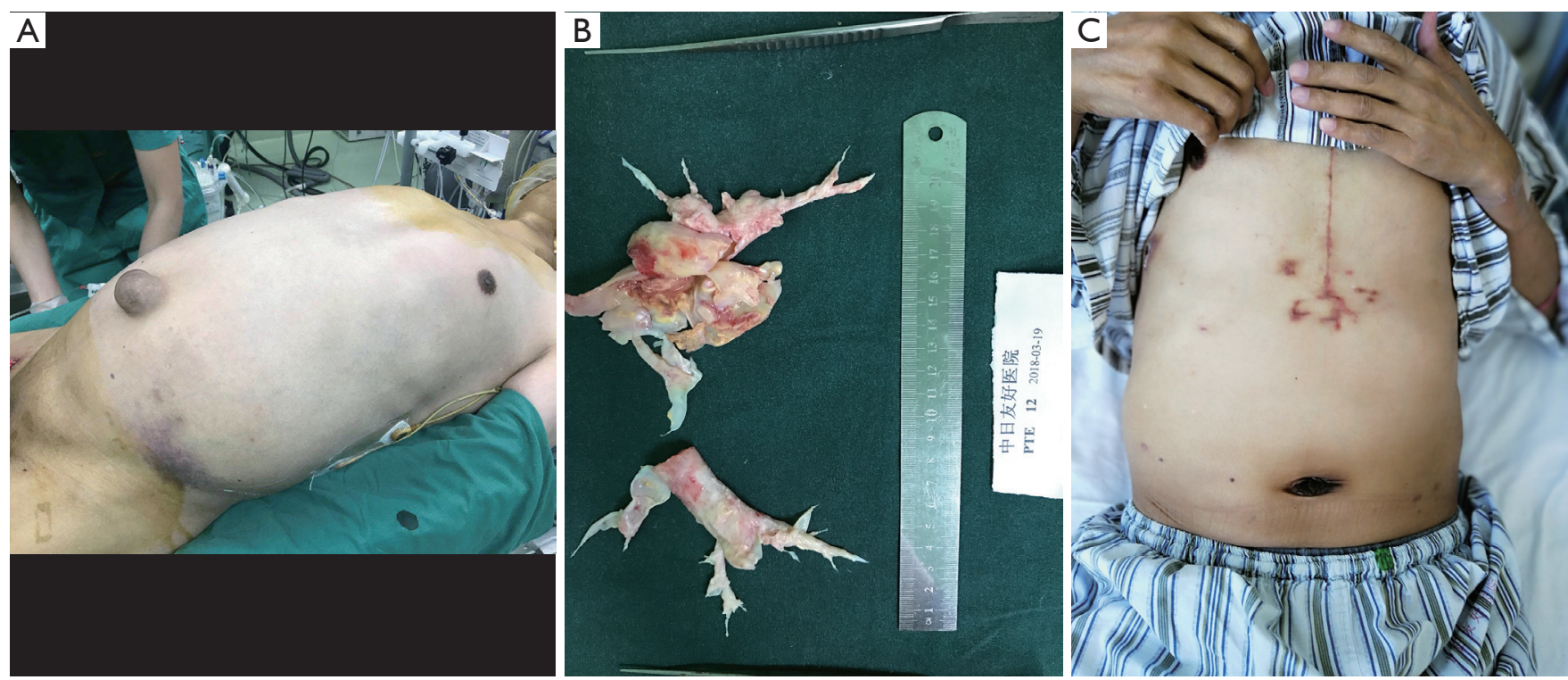

Figure 3 A typical case of CTEPH patient underwent PTE surgery. (A) Abdominal bulging in a patient with end-stage pulmonary hypertension; (B) thickened intima and organized thrombus in the pulmonary artery; (C) abdominal bulging was relieved after the operation. CTEPH, chronic thromboembolic pulmonary hypertension; PTE, pulmonary thromboendarterectomy.

right atrium). In our study, all patients presented with echocardiography that was proven as TR, among whom 22 were classified as moderate or severe. As reported, in end-stage CTEPH, hepatosplenomegaly and ascites may occur due to severe TR and right heart failure (Figure 3).

Functional TR was defined as the failure of leaflet coaptation without evidence of leaflet or subvalvular apparatus abnormalities (7), usually secondary to longlasting pulmonary hypertension. Severe functional TR also usually develops in patients with left-sided valvular disease. In the past, surgeons have been conservative in performing tricuspid annuloplasty because TR could be reduced after left-sided surgery alone (14). However, more studies have shown that simultaneous tricuspid annuloplasty and leftsided heart surgery are associated with better right ventricle remodeling and long-term results (5,15-17). As for TR associated with CTEPH, there is a limited amount of data that has been published to illustrate whether simultaneous 
tricuspid annuloplasty should be performed in PTE surgery.

Sadeghi et al. (6) reported that $70 \%$ of patients showed a marked decrease in severe TR even without annuloplasty of a dilated tricuspid annulus, and the resolution of severe TR is related to the reduction of pulmonary hypertension. Menzel et al. (12) reported that $82.8 \%$ of moderate to severe TR in CTEPH patients could be alleviated after PTE without added tricuspid repair. Although the research did not specifically focus on whether tricuspid annuloplasty should be performed simultaneously, Li et al. (18) reported there to be a significant improvement in right ventricular dysfunction, including TR velocity and TR grade in patients receiving PTE after 2-year followup. Type 1 and type 2 CTEPH may be associated with better improvement of TR after PTE, in which the lesion location is more proximal $(7,13)$. Different inspection modalities have demonstrated right ventricular reverse remodeling after PTE, and the improvement of TR and pulmonary artery compliance (19-23). Thistlethwaite et al. (24) reported three simultaneous PTE and tricuspid annuloplasty for patients with structural abnormality of tricuspid valve leaflet or chordae. They suggested that tricuspid valve repair should be performed for organic TR.

The largest case series was reported by Madani (8), the mean PVR of the patients in the study was 719 and 861.2 dynes.sec $\cdot \mathrm{cm}^{-5}$ relatively. Maybe because the awareness and understanding of CTEPH is still lacking in China. Most of the patients received PTE were advancedstage CTEPH with PVR $>1,000$ dynes.sec $\cdot \mathrm{cm}^{-5}$. In our study series, the mean PVR was $1,025.4$ dynes $\cdot \mathrm{sec} \cdot \mathrm{cm}^{-5}$, all patients were functional TR. We did not conduct tricuspid annuloplasty even for severe TR. The results showed that the most severe TR could be quickly alleviated solely after PTE surgery. The current data suggested that tricuspid valve surgery need not be performed routinely in patients with CTEPH and secondary functional TR. However, long-term follow-up should be conducted to evaluate the right ventricular and tricuspid function.

There are several limitations to our study. First, the sample size is small, and it is only our preliminary experience. Second, the follow-up time is short; whether the improvement of TR will last should be further investigated.

\section{Conclusions}

In conclusion, functional TR could be alleviated after PTE even in patients with high PVR. However, the long-term results need to be further investigated.

\section{Acknowledgments}

Funding: None.

\section{Footnote}

Conflicts of Interest: The authors have no conflicts of interest to declare.

Ethical Statement: The authors are accountable for all aspects of the work in ensuring that questions related to the accuracy or integrity of any part of the work are appropriately investigated and resolved. The study procedures followed institutional guidelines and was approved by the institutional review board of our hospital. The enrolled patients have signed the informed consent for the use of the data.

Open Access Statement: This is an Open Access article distributed in accordance with the Creative Commons Attribution-NonCommercial-NoDerivs 4.0 International License (CC BY-NC-ND 4.0), which permits the noncommercial replication and distribution of the article with the strict proviso that no changes or edits are made and the original work is properly cited (including links to both the formal publication through the relevant DOI and the license). See: https://creativecommons.org/licenses/by-nc-nd/4.0/.

\section{References}

1. Ende-Verhaar YM, Cannegieter SC, Vonk Noordegraaf A, et al. Incidence of chronic thromboembolic pulmonary hypertension after acute pulmonary embolism: a contemporary view of the published literature. Eur Respir J 2017. doi: 10.1183/13993003.01792-2016.

2. Pengo V, Lensing AW, Prins $M H$, et al. Incidence of chronic thromboembolic pulmonary hypertension after pulmonary embolism. N Engl J Med 2004;350:2257-64.

3. Mahmud E, Madani MM, Kim NH, et al. Chronic Thromboembolic Pulmonary Hypertension: Evolving Therapeutic Approaches for Operable and Inoperable Disease. J Am Coll Cardiol 2018;71:2468-86.

4. Rahnavardi M, Yan TD, Cao C, et al. Pulmonary thromboendarterectomy for chronic thromboembolic pulmonary hypertension: a systematic review. Ann Thorac Cardiovasc Surg 2011;17:435-45.

5. Chikwe J, Itagaki S, Anyanwu A, et al. Impact of Concomitant Tricuspid Annuloplasty on Tricuspid Regurgitation, Right Ventricular Function, and Pulmonary 
Artery Hypertension After Repair of Mitral Valve Prolapse. J Am Coll Cardiol 2015; 65:1931-8.

6. Sadeghi HM, Kimura BJ, Raisinghani A, et al. Does lowering pulmonary arterial pressure eliminate severe functional tricuspid regurgitation? Insights from pulmonary thromboendarterectomy. J Am Coll Cardiol 2004;44:126-32.

7. Thistlethwaite PA, Jamieson SW. Tricuspid valvular disease in the patient with chronic pulmonary thromboembolic disease. Curr Opin Cardiol 2003;18:111-6.

8. Madani MM, Auger WR, Pretorius V, et al. Pulmonary endarterectomy: recent changes in a single institution's experience of more than 2,700 patients. Ann Thorac Surg 2012;94:97-103; discussion 103.

9. Rudski LG, Lai WW, Afilalo J, et al. Guidelines for the echocardiographic assessment of the right heart in adults: a report from the American Society of Echocardiography endorsed by the European Association of Echocardiography, a registered branch of the European Society of Cardiology, and the Canadian Society of Echocardiography. J Am Soc Echocardiogr 2010;23:685713; quiz 786-8.

10. Menzel T, Wagner S, Kramm T, et al. Pathophysiology of impaired right and left ventricular function in chronic embolic pulmonary hypertension: changes after pulmonary thromboendarterectomy. Chest 2000;118:897-903.

11. Delcroix M, Vonk Noordegraaf A, Fadel E, et al. Vascular and right ventricular remodelling in chronic thromboembolic pulmonary hypertension. Eur Respir J 2013; 41:224-32.

12. Menzel T, Kramm T, Wagner S, et al. Improvement of tricuspid regurgitation after pulmonary thromboendarterectomy. Ann Thorac Surg 2002;73:756-61.

13. Thistlethwaite PA, Mo M, Madani MM, et al. Operative classification of thromboembolic disease determines outcome after pulmonary endarterectomy. J Thorac Cardiovasc Surg 2002;124:1203-11.

14. Braunwald NS, Ross J, Morrow AG. Conservative management of tricuspid regurgitation in patients undergoing mitral valve replacement. Circulation 1967;35:I63-9.

15. Navia JL, Nowicki ER, Blackstone EH, at al. Surgical management of secondary tricuspid valve regurgitation: annulus, commissure, or leaflet procedure? J Thorac Cardiovasc Surg 2010;139:1473-82.e5.

16. Kim JB, Yoo DG, Kim GS, et al. Mild-to-moderate functional tricuspid regurgitation in patients undergoing valve replacement for rheumatic mitral disease: the influence of tricuspid valve repair on clinical and echocardiographic outcomes. Heart 2012;98:24-30.

17. Sakata T, Mogi K, Sakurai M, et al. Effect of tricuspid annuloplasty concomitant with left heart surgery on right heart geometry and function. J Thorac Cardiovasc Surg 2018;156:1050-61.

18. Li YD, Zhai Z, Wu Y, et al. Improvement of right ventricular dysfunction after pulmonary endarterectomy in patients with chronic thromboembolic pulmonary hypertension: utility of echocardiography to demonstrate restoration of the right ventricle during 2-year follow-up. Thromb Res 2013;131:e196-201.

19. Berman M, Gopalan D, Sharples L, et al. Right ventricular reverse remodeling after pulmonary endarterectomy: magnetic resonance imaging and clinical and right heart catheterization assessment. Pulm Circ 2014;4:36-44.

20. Maeba H, Nakatani S, Sugawara M, et al. Different time course of changes in tricuspid regurgitant pressure gradient and pulmonary artery flow acceleration after pulmonary thromboendarterectomy: implications for discordant recovery of pulmonary artery pressure and compliance. Circ J 2007;71:1771-5.

21. Chow LC, Dittrich HC, Hoit BD, et al. Doppler assessment of changes in right-sided cardiac hemodynamics after pulmonary thromboendarterectomy. Am J Cardiol 1988;61:1092-7.

22. Wong DJ, Sampat U, Gibson MA, et al. Tricuspid annular plane systolic excursion in chronic thromboembolic pulmonary hypertension before and after pulmonary thromboendarterectomy. Echocardiography 2016;33:1805-9.

23. Casaclang-Verzosa G, McCully RB, Oh JK, et al. Effects of pulmonary thromboendarterectomy on right-sided echocardiographic parameters in patients with chronic thromboembolic pulmonary hypertension. Mayo Clin Proc 2006;81:777-82.

24. Thistlethwaite PA, Auger WR, Madani MM, et al. Pulmonary thromboendarterectomy combined with other cardiac operations: indications, surgical approach, and outcome. Ann Thorac Surg 2001;72:13-7; discussion 17-9.

Cite this article as: Zhen Y, Zhang J, Liu X, Sun G, Zheng X, Han Y, Zhai Z, Li A, Lin F, Liu P. Impact of pulmonary thromboendarterectomy on tricuspid regurgitation in patients with chronic thromboembolic pulmonary hypertension: a single-center prospective cohort experience. J Thorac Dis 2020;12(3):758-764. doi: 10.21037/jtd.2019.12.99 